\title{
Robustaside B and para-hydroxyphenol: Phenolic and antioxidant compounds purified from Cnestis ferruginea D.C induced membrane permeability transition in rat liver mitochondria
}

\author{
RAHMAT A. ADISA ${ }^{1,2}$ and OLUFUNSO O. OLORUNSOGO ${ }^{1}$ \\ ${ }^{1}$ Laboratories for Biomembrane Research and Biotechnology, \\ Department of Biochemistry, College of Medicine, University of Ibadan, Ibadan 200284, Nigeria
}

Received March 4, 2013; Accepted July 1, 2013

DOI: $10.3892 / \mathrm{mmr} .2013 .1674$

\begin{abstract}
The antioxidant properties of robustaside B and para-hydroxyphenol isolated from Cnestis ferruginea were measured as the rate of inhibition of thiobarbituric acid reactive substance (TBARS) production in the $\mathrm{Fe}^{2+}$ /ascorbate system. The modulatory effects of the compounds on mitochondrial membrane permeability transition (MMPT) were monitored spectrophotometrically as decreases in light scattering at $540 \mathrm{~nm}$. The varying concentrations of robustaside B and para-hydroxyphenol $(0.05,0.1,0.2,0.25,0.5,0.75$ and $1 \mathrm{mM})$ significantly reduced $(\mathrm{P}<0.05)$ the amount of TBARS generated by the $\mathrm{Fe}^{2+}$ /ascorbate system by $85.3,86.4,86.0$, $86.1,86.0,86.0$ and $86.0 \%$ and 86.7, 81.3, 81.3, 80, 80, 82.6 and $83.1 \%$, respectively. Similarly, quercetin, a standard antioxidant, was found to induce an $80 \%$ reduction in the amount of TBARS produced. The same $\mathrm{IC}_{50}$ value of $0.025 \mathrm{mM}$ was observed for robustaside B, para-hydroxyphenol and quercetin. Pre-incubation of varying concentrations of robustaside $B$ $(0.125,0.2,0.5$ and $1 \mathrm{mM})$ with succinate-energized mitochondria induced MMPT pore opening by $0,-33.3,-59.3$ and $-218.5 \%$, compared with control mitochondria. Para-hydroxyphenol at $0.1,0.2,0.25$ and $0.5 \mathrm{mM}$ induced MMPT pore opening in a concentration-dependent manner up to $0.25 \mathrm{mM}$ by $-21,-54.4$ and $-107.0 \%$, respectively. Quercetin at $0.05,0.1,0.25,0.5,0.75$ and $1 \mathrm{mM}$ also induced MMPT pore opening in the absence of calcium in a concentration-dependent manner by $5,3.7,-42.6$,
\end{abstract}

Correspondence to: Dr Rahmat A. Adisa, Laboratories for Biomembrane Research and Biotechnology, Department of Biochemistry, College of Medicine, University of Ibadan, Ibadan 200284, Nigeria

E-mail: radisa22@yahoo.com; radisa@unilag.edu.ng

Present address: ${ }^{2}$ Laboratories for Biomembrane and Cancer Research, Department of Biochemistry, College of Medicine, University of Lagos, P.M.B. 12003, Idi-araba, Lagos 100254, Nigeria

Key words: mitochondrial permeability transition, lipid peroxidation, mitochondrial swelling, para-hydroxyphenol, robustaside $\mathrm{B}$, quercetin
$-81.5,-187$ and $-161.1 \%$, respectively. The current observations confirm the antioxidant properties of robustaside B and para-hydroxyphenol, and indicate a potential therapeutic use of the compounds for the treatment of diseases requiring the induction of cell death, including cancer.

\section{Introduction}

Phytochemicals, including polyphenolic compounds, are well studied chemopreventive agents in the development of cancer. These compounds, including flavonoids, occur ubiquitously in foods of plant origin and are consumed daily in the majority of Western diets (1). Flavonoids are subcategorized into flavonols, flavones, catechins, flavanones, chalcones, anthocyanidins and isoflavonoids. Flavonoids exhibit antioxidant properties by inhibiting lipid peroxidation induced by various pro-oxidants in liver homogenate, microsomes, mitochondria and liposomes (2). Free radical-mediated lipid peroxidation has been grossly implicated in the pathogenesis of diseases, including cancer, atherosclerosis, neurological disorders and toxic cell injury (3-5), as radicals react with critical cellular components, such as DNA, lipids and proteins. The antioxidant activities of polyphenols have been observed to be associated with the ability of polyphenols to chelate metal ions and to scavenge singlet oxygen, superoxide anions, peroxyl radicals, hydroxyl radicals and peroxynitrite $(2,6)$, as well as inhibit lipid peroxidation (7). Thus, polyphenols with antioxidant activities may foster useful health maintenance protection to cells against damage caused by reactive oxygen species.

The phenolic compounds, robustaside B (6'-3", 4"-dihydroxycinnamoyl), an arbutin derivative $(8,9)$, and para-hydroxyphenol (Fig. 1), have been isolated for the first time (10) from leaves of Cnestis ferruginea (Connaraceae). This plant is commonly found in West Africa and is known for its use as a laxative, antimicrobial agent and treatment of tooth cavities (11). Arbutins are known for antibacterial and diuretic activities as well as inhibition of melanin biosynthesis and are therefore raw materials in cosmetic industries (12). This class of compounds has been reported in the leaves of Grevillea robusta (8). The chemical structure of robustaside B and para-hydroxyphenol indicates the presence of phenolic moieties which have been shown to scavenge DPPH radicals (10). 
According to the WHO in 2009 (13), cancer was the third most common cause of mortalities worldwide, particularly in developed countries, with 12.7 million new cases diagnosed and 7.6 million cancer-related mortalities occurring in 2008 (14). Numerous anticancer drugs used for chemotherapy aim to prolong the life of the patient. A number of these anticancer agents originate in plants, including camptothecins, vincristine and irinotecan, and also in microorganisms, such as doxorubicin, dactinomycin, mitomycin and bleomycin (15). At present, anticancer drugs are highly cytotoxic to normal cells causing unpleasant side-effects to patients and eventually having reduced therapeutic efficacy due to drug resistance (16). Therefore, there has been a shift in research attention into the development of efficient, selective and less toxic anticancer drugs.

The induction of apoptosis is an inexhaustible mechanism for combating and gradually eliminating cancer cells. Apoptosis may be mediated via the extrinsic death receptor and intrinsic mitochondrial-mediated pathways (17). The mitochondrial-mediated pathway involves the opening of a non-specific pore in the inner mitochondrial membrane known as the mitochondrial permeability transition pore (MPT). Mitochondrial permeability transition is a phenomenon caused by calcium overload, oxidative stress or cellular insult, which leads to massive swelling and depolarization of the mitochondria, depletion of pyrimidine nucleotides, release of cytochrome $c$ and other apoptotic factors $(18,19)$, uncoupling of oxidative phosphorylation, hydrolysis of ATP by mitochondrial $\mathrm{F}_{0} \mathrm{~F}_{1}$ ATPases and cell death $(20,21)$. A number of phytochemicals, including polyphenols, have been observed to function as chemopreventive agents in the development of cancer and diseases resulting from dysregulated apoptosis, including autoimmune diseases and spreading of viral infections $(22,23)$. To investigate the incidence of cancer, these phytochemicals and plant extracts are being targeted at mechanisms of apoptosis involving regulatory pathways. Pathways, including the Bcl-2 family of proteins, induction of mitochondrial swelling and dissipation of membrane potential, modulation of caspase activation and suppression, as well as induction of cytochrome $c$ release (24-26), are being examined. There is a lack of information on the pharmacological importance of robustaside B and para-hydroxyphenol beyond their radical scavenging potential (10). Thus, it has become imperative to establish the antioxidant properties of these compounds, in particular, the anti-lipid peroxidative properties and modulatory effects on membrane permeability transition pore opening in rat liver mitochondria.

\section{Materials and methods}

Chemicals and reagents. Robustaside B and para-hydroxyphenol were previously purified from the leaves of Cnestis ferruginea and characterized in our laboratory. Quercetin, spermine, HEPES, D-mannitol and thiobarbituric acid were purchased from Sigma-Aldrich (St. Louis, MO, USA). Other reagents used were of analytical grade.

Animals. Healthy male Wistar strain albino rats, weighing between 150 and $200 \mathrm{~g}$, were purchased from the Animal House of the Department of Biochemistry, University of
Ibadan (Ibadan, Nigeria). Animals were handled according to the NIH regulations guiding animal handling (ensured by the Department of Biochemistry Postgraduate Programme Board of the University of Ibadan, Ibadan, Nigeria) and were kept under standard conditions of light/dark cycles and a temperature of $23 \pm 2^{\circ} \mathrm{C}$. Animals were supplied with water and fed ad libitum throughout the duration of the experiment.

Preparation of low ionic strength mitochondria. Rats were sacrificed by cervical dislocation and livers were excised, rinsed with buffer $\mathrm{C}$ [210 $\mathrm{mM}$ mannitol, $70 \mathrm{mM}$ sucrose, $5 \mathrm{mM}$ HEPES-KOH and EGTA (1 $\mathrm{mM}, \mathrm{pH}$ 7.4)], blotted, weighed, minced and homogenized in a glass Teflon homogenizer to produce a $10 \%$ suspension. The homogenate was subjected to differential centrifugation to prepare low ionic strength mitochondria according to the method described by Johnson and Lardy (27). The homogenate was spun twice at $885 \mathrm{x} \mathrm{g}$ for $5 \mathrm{~min}$ and mitochondria were centrifuged at 5,000 x $\mathrm{g}$ for $20 \mathrm{~min}$ in an Angle 13 refrigerated centrifuge (MSE, London, UK). Mitochondria were washed twice with buffer D [210 mM mannitol, $70 \mathrm{mM}$ sucrose, 5\% BSA (pH 7.4)]. Mitochondrial pellets were suspended in MSH buffer [210 $\mathrm{mM}$ mannitol, $70 \mathrm{mM}$ sucrose and $5 \mathrm{mM}$ HEPES-KOH (pH 7.4)] to produce a mitochondrial suspension $(1 \mathrm{ml})$ that is the equivalent to mitochondria isolated from $1 \mathrm{~g}$ tissue.

Determination of protein concentration. Mitochondrial protein concentrations were determined according to the method previously described by Lowry et al (28) using BSA as the standard.

Antioxidant studies. Inhibition of lipid peroxidation in mitochondria was determined spectrophotometrically by measuring the intensity of the pink color of thiobarbituric acid reactive substances (TBARS) induced in the $\mathrm{Fe}^{2+} /$ ascorbate system at $532 \mathrm{~nm}$ according to the method described by Varshney and Kale (29). The reaction mixture contained mitochondria $(0.12 \mathrm{mg} / \mathrm{ml})$ in Tris- $\mathrm{HCl}(30 \mathrm{mM})$, ferrous ammonium sulphate $(0.16 \mathrm{mM})$, ascorbic acid $(0.06 \mathrm{mM})$ and various concentrations of the compounds $(0.05-1 \mathrm{mM})$ in a final reaction volume of $0.5 \mathrm{ml}$, as previously described (30) and was incubated for $1 \mathrm{~h}$ at $37^{\circ} \mathrm{C}$. The resulting TBARS were measured spectrophotometrically. The reaction mixture $(0.4 \mathrm{ml})$ was mixed with $1.6 \mathrm{ml}$ Tris- $\mathrm{HCl}$ buffer $(0.15 \mathrm{M})$ to which $0.5 \mathrm{ml}$ trichloroacetic acid $(30 \%)$ was added (to terminate the reaction). Thereafter, $0.5 \mathrm{ml}$ thiobarbituric acid $(0.75 \%)$ was added and placed in a water bath for $30 \mathrm{~min}$ at $95^{\circ} \mathrm{C}$, cooled on ice and centrifuged at room temperature for $10 \mathrm{~min}$ at 3,000 rpm in an SM902B benchtop centrifuge (Surgifriend Medicals, Middlesex, UK). Absorbance of the clear pink supernatant was measured against a reference blank of distilled water at $532 \mathrm{~nm}$ using a Camspec 106 spectrophotometer (Spectronic Camspec Ltd., Garforth, UK).

Determination of mitochondrial swelling. The extent of mitochondrial swelling was utilized as a measure of MPT pore opening in the presence or absence of calcium, the triggering agent, according to the method described by Lapidus and Sokolove (31). Briefly, mitochondria $(0.12 \mathrm{mg} / \mathrm{ml})$ were preincu- 
<smiles>O=C(/C=C/c1ccc(O)c(O)c1)OCC1CC(Oc2ccc(O)cc2)C(O)C(O)C1O</smiles>

B<smiles>Oc1ccccc1</smiles>

Figure 1. Structure of (A) robustaside B and (B) para-hydroxyphenol isolated from leaves of Cnestis ferruginea (D.C).

bated with $0.8 \mu \mathrm{M}$ rotenone in MSH buffer [210 $\mathrm{mM}$ mannitol, $70 \mathrm{mM}$ sucrose, $5 \mathrm{mM}$ HEPES-KOH ( $\mathrm{pH} 7.4)$ ] for $3.5 \mathrm{~min}$, following which, $5 \mathrm{mM}$ succinate was added to energize the reaction in a total reaction volume of $1.25 \mathrm{ml}$ in the absence of calcium chloride. For estimation of calcium-induced MPT pore opening, mitochondria $(0.12 \mathrm{mg} / \mathrm{ml})$ were incubated for $3 \mathrm{~min}$ with rotenone in $\mathrm{MSH}$ buffer and $300 \mathrm{nmol} \mathrm{CaCl} \mathrm{Cl}_{2} \cdot 2 \mathrm{H}_{2} \mathrm{O}$ was added immediately to trigger swelling. In addition, $5 \mathrm{mM}$ succinate was added $30 \mathrm{sec}$ later. To assess the effects of spermine $(0.1 \mathrm{mM})$, robustaside $\mathrm{B}$ or para-hydroxyphenol (0.1-1 mM), mitochondria were pre-incubated with rotenone, spermine or the compounds for $3 \mathrm{~min}$ in MSH buffer. Calcium chloride was added to the reaction medium at $3 \mathrm{~min}$ and 30 seconds later, sodium succinate was added to energize the reaction. To determine the effects of the compounds alone on mitochondrial swelling, mitochondria were pre-incubated with rotenone and the pure compounds for $3.5 \mathrm{~min}$ in $\mathrm{MSH}$ buffer. During the incubation, sodium succinate was added at $3.5 \mathrm{~min}$ and the rate of mitochondrial swelling was estimated spectrophotometrically as a decrease in absorbance at $540 \mathrm{~nm}$ and was measured every $30 \mathrm{sec}$ for $12 \mathrm{~min}$.

Statistical analysis. Data were analyzed using ANOVA and are expressed as the mean $\pm \mathrm{SD}$. $\mathrm{P}<0.05$ was considered to indicate a statistically significant difference.

\section{Results}

Antioxidantactivity ofRobustaside B and para-hydroxyphenol. Comparative effects of robustaside B, para-hydroxyphenol and quercetin on $\mathrm{Fe}^{2+} /$ ascorbate-induced mitochondrial membrane lipid peroxidation are presented in Fig. 2. Robustaside B decreased the amount of TBARS produced during lipid peroxidation induced by the $\mathrm{Fe}^{2+}$ /ascorbate system in rat liver mitochondria in vitro in a concentration-dependent manner. Varying concentrations of robustaside B $(0.05,0.1,0.2,0.25$, $0.5,0.75$ and $1 \mathrm{mM})$ significantly $(\mathrm{P}<0.05)$ reduced the amount of TBARS generated by $85.3,86.4,86.0,86.1,86.0$, 86.0 and $86.0 \%$, respectively. Similarly the same concentrations of para-hydroxyphenol significantly $(\mathrm{P}<0.05)$ decreased the amount of TBARS by $86.7,81.3,81.3,80,80,82.6$ and $83.1 \%$, respectively. In addition, similar concentrations of

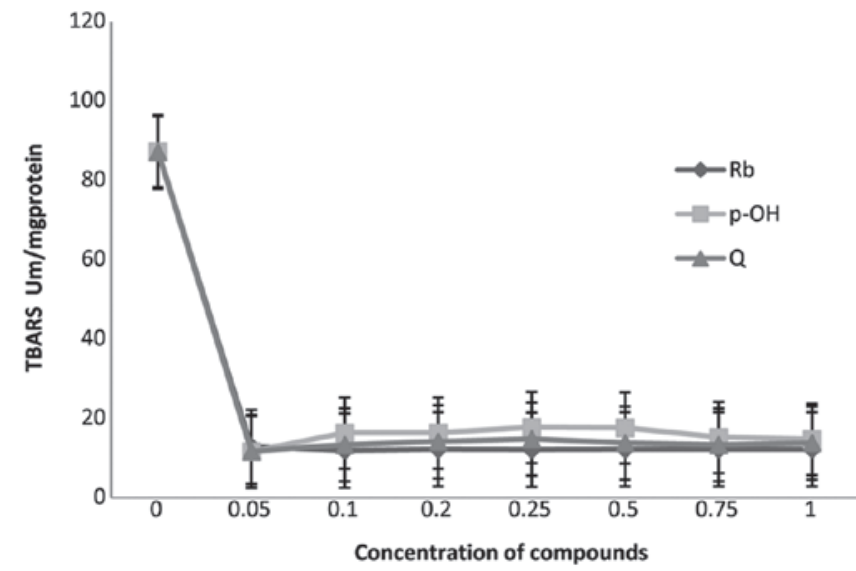

Figure 2. Effects of $\mathrm{Rb}$ and $\mathrm{pOH}$ on $\mathrm{Fe}^{2+} /$ ascorbate-induced rat liver mitochondrial membrane lipid peroxidation. $\mathrm{Rb}$, robustaside $\mathrm{B} ; \mathrm{pOH}$, para-hydroxyphenol; Q, quercetin; TBARS, thiobarbituric acid reactive substances.

quercetin, an antioxidant used as the standard, significantly $(\mathrm{P}<0.05)$ reduced the amount of TBARS produced by 86.6 , 84.8, 83.8, 83.0, 84.2, 84.8 and 84.2\%, respectively (Fig. 2). The observations indicated that there was no significant difference $(\mathrm{P}>0.05)$ in the degree of inhibition of membrane lipid peroxidation by all three compounds. All compounds elicited at least an $80 \%$ reduction in the amount of TBARS and the same $\mathrm{IC}_{50}$ value of $0.025 \mathrm{mM}$.

Effects of Robustaside B on MPT pore opening with or without $\mathrm{CaCl}_{2}$. Pre-incubation of varying concentrations of robustaside $\mathrm{B}(0.125,0.25,0.5$ and $1 \mathrm{mM})$ with mitochondria energized by succinate induced MPT pore opening in a concentration-dependent manner by $0,-33.3,-59.3$ and $-218.5 \%$, respectively, as compared with control untreated mitochondria in the absence of calcium (Fig. 3). In the presence of calcium, similar concentrations of robustaside B decreased the extent of MPT pore opening in a concentration-dependent manner but in the reverse order. For example, 0.125, 0.25, 0.5 and $1.0 \mathrm{mM}$ robustaside $\mathrm{B}$ protected the mitochondrial membrane from calcium-induced MPT by 60.3, 73.3, 27.6 and 19.8\%, respectively (Fig. 4). The maximum inhibitory concentration of robustaside B obtained was $0.25 \mathrm{mM}$ following which the extent of inhibition decreased as the concentration increased. There was a negative correlation observed between the inhibition of lipid peroxidation and induction of mitochondrial membrane permeability transition (MMPT) pore opening in the presence or absence of robustaside B (absence of calcium, $\mathrm{r}=-0.3$; presence of calcium, $\mathrm{r}=-0.834$ ).

Induction of MPT pore opening with or without $\mathrm{CaCl}_{2}$ by para-hydroxyphenol. By contrast, in the absence of calcium, varying concentrations of para-hydroxyphenol $(0.1,0.2$, 0.25 and $0.5 \mathrm{mM}$ ) induced MPT pore opening in a concentration-dependent manner up to $0.25 \mathrm{mM}$ by $-21,-54.4$ and $-107.0 \%$, respectively (Fig. 5). Similarly, in the presence of calcium, the same concentrations of para-hydroxyphenol further induced MPT pore opening by 78.9, -261, -261 and $-213.3 \%$, respectively, when compared with induction by calcium alone. As the concentration of the compound 

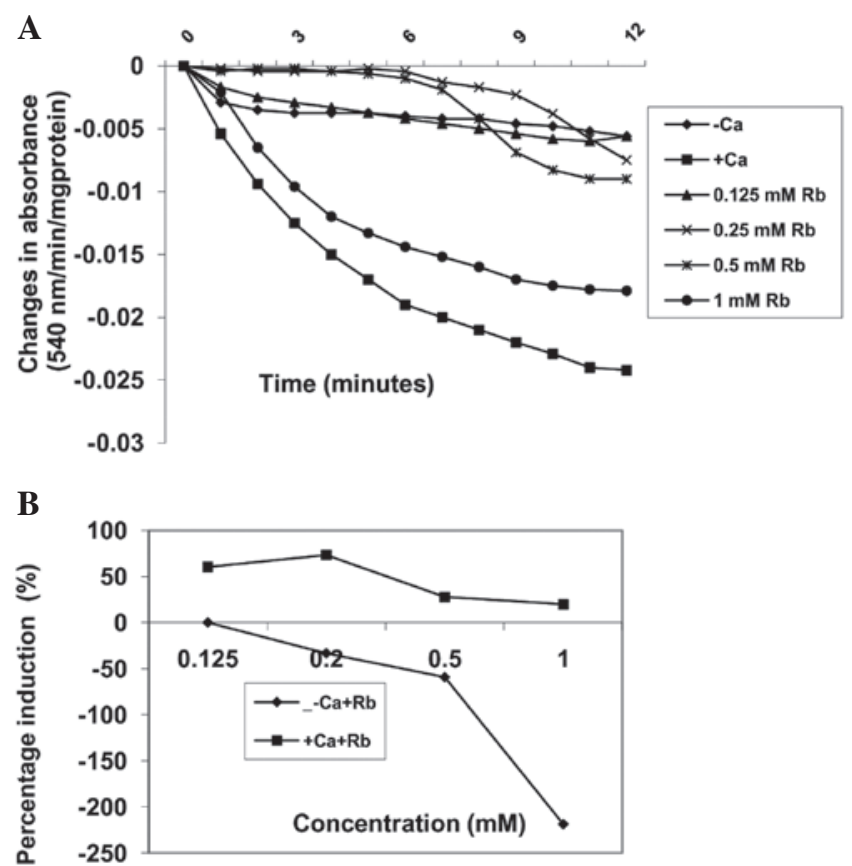

Figure 3. (A) Induction of MPT by Rb in normal rat liver mitochondria in the absence of calcium. (B) Comparison of inhibition and induction of MPT pore opening by $\mathrm{Rb}$ in the absence and presence of calcium. $\mathrm{Rb}$, robustaside $\mathrm{B}$; MPT, mitochondrial permeability transition.

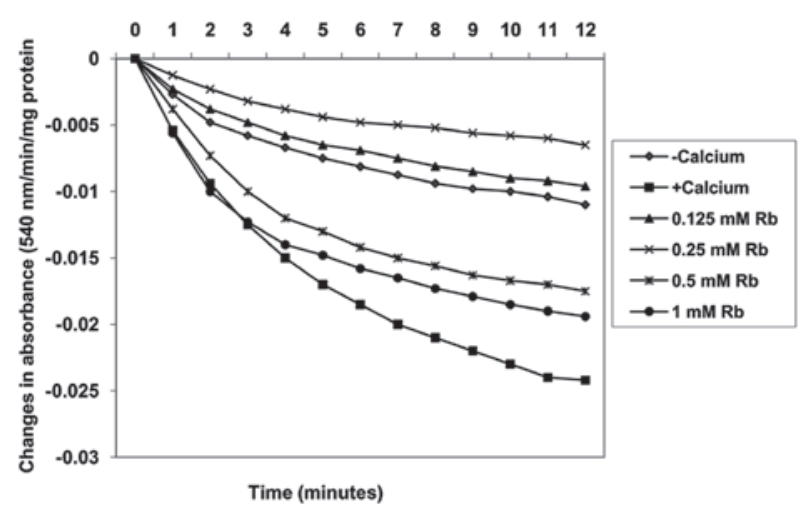

Figure 4. Inhibition of calcium-induced membrane permeability transition by $\mathrm{Rb}$ in normal rat liver mitochondria energized by succinate. Rb, robustaside $\mathrm{B}$.

increased, the extent of pore opening also increased (Fig. 6). In addition, there was a positive correlation observed between the inhibition of lipid peroxidation and the induction of MMPT pore opening in the presence or absence of calcium by para-hydroxyphenol (absence of calcium, $r=0.437$; presence of calcium, $\mathrm{r}=0.408$ ).

Effects of quercetin on MPT pore opening with or without $\mathrm{CaCl}_{2}$. Varying concentrations of quercetin $(0.05,0.1,0.25$, $0.5,0.75$ and $1 \mathrm{mM}$ ) also induced MPT pore opening in the absence of calcium in a concentration-dependent manner by 5, 3.7, -42.6, -81.5, -187 and $-161.1 \%$, respectively (Fig. 7). By contrast, the same concentrations of quercetin decreased MPT pore opening triggered by calcium by $-1.0,-1.0,42.9,51.0,54$ and $54 \%$, respectively (Fig. $8 \mathrm{~A}$ ). There was a positive correla-

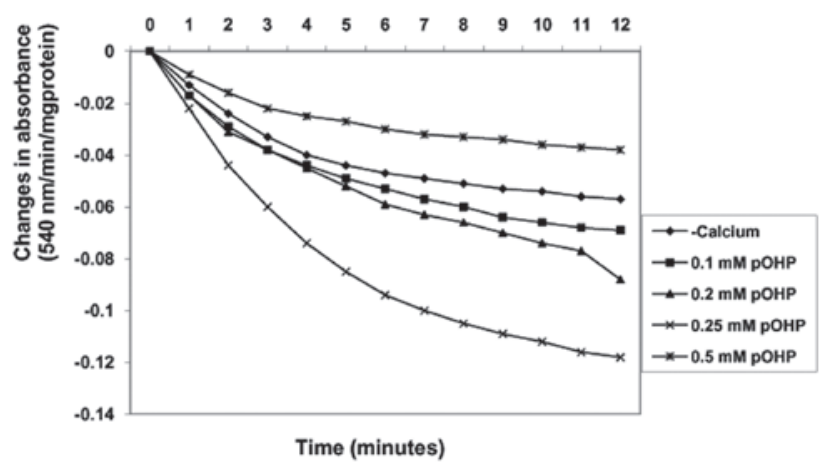

Figure 5. Induction of mitochondrial membrane permeability transition by p-OHP in the absence of calcium. $p$-OHP, para-hydroxyphenol.

A

$\begin{array}{lllllllllllll}0 & 1 & 2 & 3 & 4 & 5 & 6 & 7 & 8 & 9 & 10 & 11 & 12\end{array}$

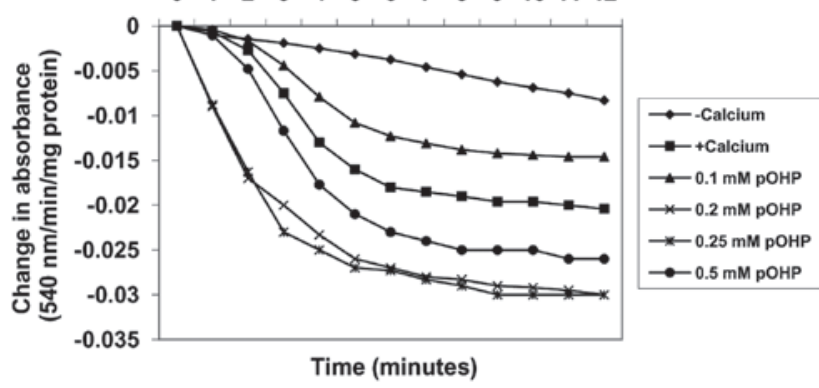

B

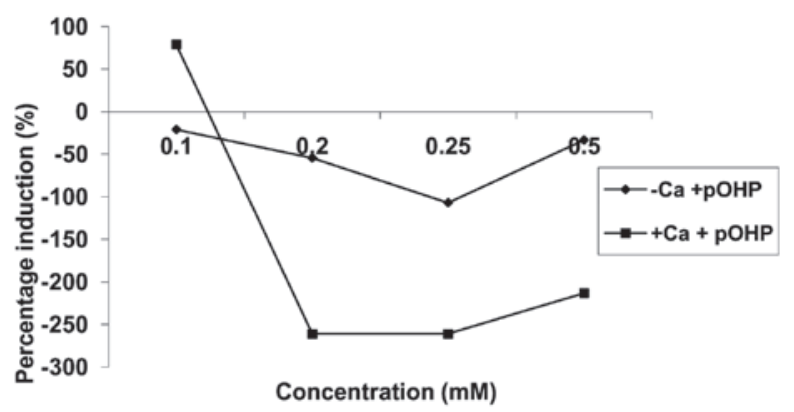

Figure 6. (A) Induction of calcium-induced MPT by $p$-OHP in normal rat liver mitochondria energized by succinate. (B) Induction of MPT by $p$-OHP in the presence and absence of calcium. $p$-OHP, para-hydroxyphenol; MPT, mitochondrial permeability transition.

tion between the inhibition of lipid peroxidation and induction of mitochondrial permeability transition pore opening by quercetin. By contrast, the inhibition of lipid peroxidation was negatively correlated with inhibition of mitochondrial permeability transition pore opening by quercetin.

\section{Discussion}

Research is currently directed towards the search for novel anticancer agents from natural sources which are physiologically non-toxic and inert to normal cells. Numerous traditionally used herbal remedies are being subjected to modern purification processes for the characterization of active components for possible therapeutic purposes against human cancers (32-34). Robustaside B and para-hydroxyphenol were purified for the first time from the leaves of Cnestis ferruginea and identified 


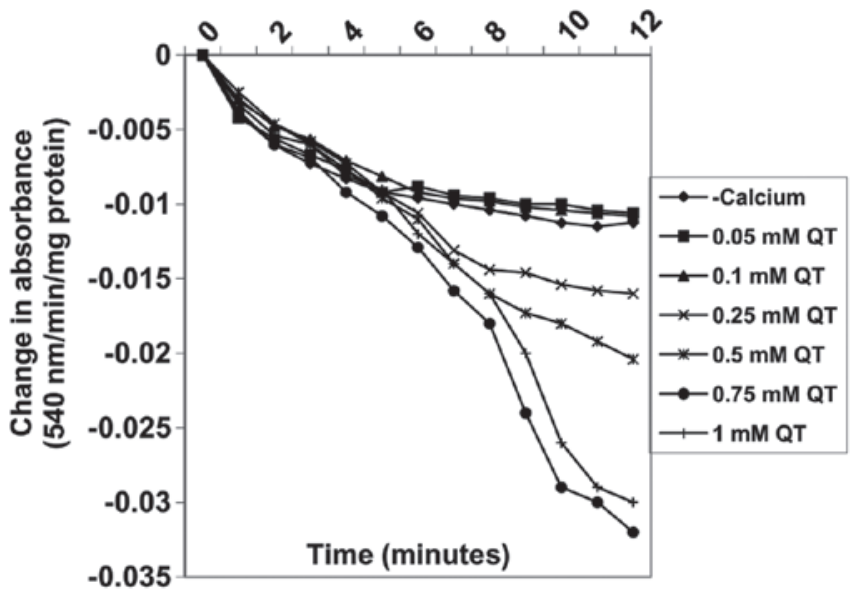

Figure 7. Induction of mitochondrial membrane permeability transition pore opening by QT in normal rat liver mitochondria energized by succinate in the absence of calcium. QT, quercetin.

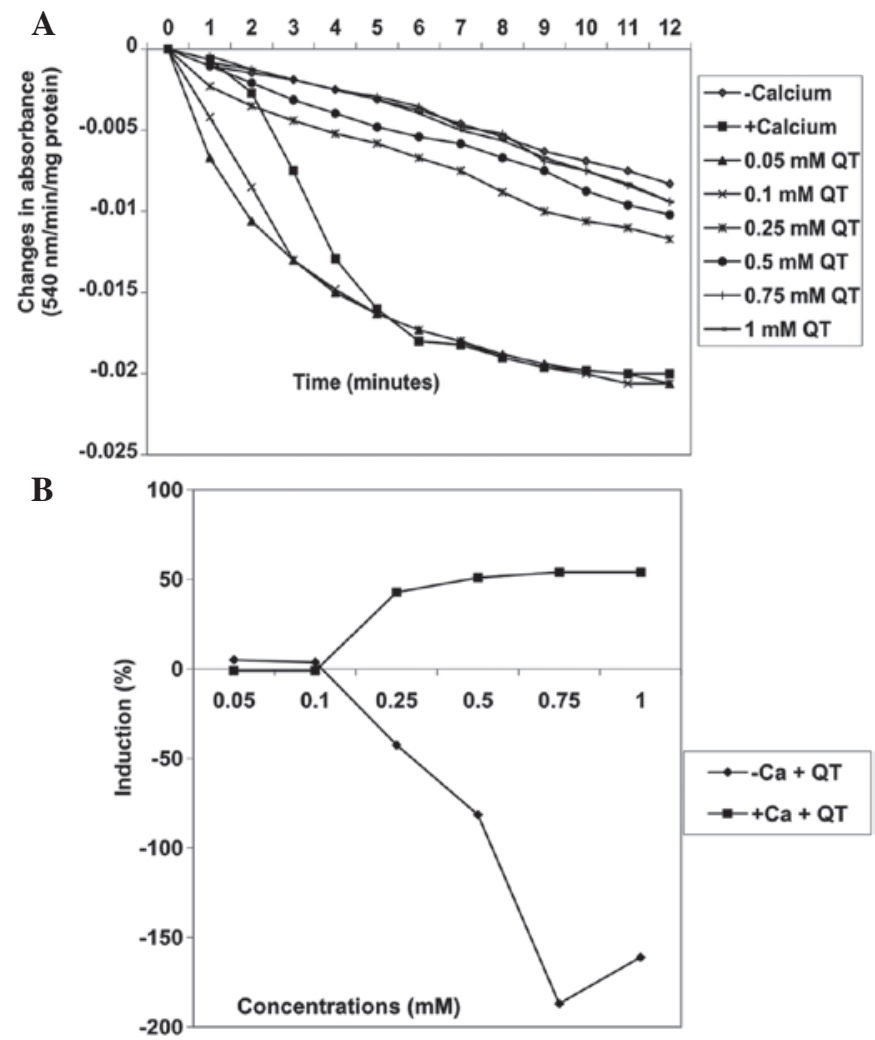

Figure 8. (A) Inhibition of calcium-induced membrane permeability transition by QT in normal rat liver mitochondria energized by succinate. (B) Comparison of induction and inhibition by QT in the absence and presence of calcium, respectively. QT, quercetin.

as radical scavengers (10). In the current study, the anti-lipid peroxidation activity of these compounds and their modulatory activity on mitochondrial membrane permeability pore opening in rat liver was reported.

Robustaside B (0.1 mM) and para-hydroxyphenol (0.05 mM) significantly $(\mathrm{P}<0.05)$ inhibited mitochondrial lipid peroxidation induced by the $\mathrm{Fe}^{2+}$ /ascorbate system by causing a significant decrease (86.4 and 86.7\%), respectively, in the amount of TBARS released by the system. There was no significant difference
( $\mathrm{P}>0.05)$ observed between the percentage inhibitions by these compounds and $0.05 \mathrm{mM}$ quercetin (86.6\%). In addition, the $\mathrm{IC}_{50}$ values $(0.025 \mathrm{mM})$ for robustaside $\mathrm{B}$, para-hydroxyphenol and quercetin also clearly revealed the similarity in their potential to inhibit lipid peroxidation stimulated in mitochondrial membranes by the $\mathrm{Fe}^{2+} /$ ascorbate system. The current observations indicate that robustaside B and para-hydroxyphenol have strong and similar antioxidant potentials comparable to that of quercetin, attributable to the presence of phenolic moieties involved in the Fenton and Haber-Weiss reactions (35) of the lipid peroxidation system. These results further support our earlier study on the strong radical scavenging properties of the compounds (10).

MMPT, measurable by mitochondrial swelling, is one of the indices of evaluating apoptosis induced through the mitochondrial pathway (36). The present observations indicated that robustaside $\mathrm{B}$, pre-incubated with isolated mitochondria from rat liver, induced MPT pore opening in a concentration-dependent manner in the absence of calcium. However, in the presence of calcium, robustaside B inhibited calcium-induced MPT pore opening with a maximum inhibitory concentration of $0.25 \mathrm{mM}$. At higher concentrations, inhibition was decreased in a concentration-dependent manner. Notably, this dual activity of robustaside $\mathrm{B}$ at different concentrations may be beneficial, in that high concentrations of robustaside $B(0.5-1 \mathrm{mM})$ may be targeted at the induction of apoptosis in proliferating cells. Following a curative period or effect, the concentration of the compound may be gradually decreased, to below $0.5 \mathrm{mM}$, before total drug withdrawal from the patient. This effect at low concentrations may also be a useful chemotherapy for patients following surgery. In addition, patients presenting with pathological conditions resulting from excessive apoptosis, including sarcopenia and HIV, may find robustaside B effective at low doses to gradually reduce apoptosis and replenish cell populations (26).

The inhibitory activity of robustaside $\mathrm{B}$ in the presence of calcium may be due to the compound mopping up calcium ions by coordination of the calcium ions to oxygen atoms in its structure. This reaction, if sustained, reduces the concentration of free calcium ions available for induction of pore opening and thus, encourages pore closure as observed at low $(0.125-0.25 \mathrm{mM})$ concentrations of robustaside B. At higher concentrations of robustaside $\mathrm{B}$, once the internal milieu calcium ion concentration is almost completely consumed, the excess robustaside $\mathrm{B}$ may intercalate between the fatty acid phospholipids in the mitochondrial membrane bilayer. This interaction may lead to alterations in membrane fluidity, a leaky membrane with dissipation of mitochondrial membrane potential and uncoupling of oxidative phosphorylation, consequently leading to apoptosis.

In the presence and absence of calcium, para-hydroxyphenol significantly induced mitochondrial permeability transition pore opening in a concentration-dependent manner. In the absence of calcium, the induction of pore opening by robustaside B is greater than that of quercetin and para-hydroxyphenol. The induction of pore opening by these compounds in the presence of calcium decreases. The presence of the polyphenolic group in the structure of robustaside B may confer polarity on the membrane structure thereby rendering it leaky. This event may encourage the association of VDAC, ANT and cyclophilin D, leading to the formation of the MPT pore. The differences in 
the structure of the compounds, particularly in the phenol ring structures present in robustaside $\mathrm{B}$, indicate a structure-function correlation accounting for the strong inductive power of robustaside B compared with para-hydroxyphenol. In addition, the similarity in the pattern of induction of MPT pore opening by robustaside $\mathrm{B}$ and quercetin may be accounted for by the presence of polyhydroxyl groups in the core ring structures of the compounds. Similar to quercetin, robustaside B and para-hydroxyphenol may be of potential use in the treatment of diseases involving the induction of apoptosis, including cancer.

In conclusion, to the best of our knowledge, robustaside B and para-hydroxyphenol have been demonstrated for the first time to possess powerful antioxidant properties since they inhibit membrane lipid peroxidation stimulated by the $\mathrm{Fe}^{2+} /$ ascorbate system in a similar manner to that of quercetin, a known antioxidant. In addition, the compounds induced MPT pore opening in rat liver mitochondria and protected the MPT pore from calcium-induced opening in vitro. By contrast, these compounds also interact with the intact mitochondria to induce pore opening in the absence of calcium. Thus, robustaside B and para-hydroxyphenol may be useful pharmaceutical applications for the design of drugs for the treatment of cancer, cardiovascular diseases and muscle wasting, along with stabilization of canned food against oxidative deterioration and amelioration of oxidatively induced disorders leading to cell death. Further in depth studies targeting the compounds against cancer cell lines are required to substantiate the anticancer activity and mechanism of induction of apoptosis in cancer cells.

\section{References}

1. Critchfield JW, Welsh CJ, Phang JM and Yeh GC: Modulation of adriamycin accumulation and efflux by flavonoids in HCT-15 colon cells. Activation of P-glycoprotein as a putative mechanism. Biochem Pharmacol 48: 1437-1445, 1994.

2. Briviba $\mathrm{K}$ and Sies H: Nonenzymatic antioxidant defense systems. In: Natural Antioxidants in Human Health and Disease. Frei B (ed). Academic Press, San Diego, CA, pp107-128, 1994.

3. Halliwell B and Gutteridge JM: Role of free radicals and catalytic metal ions in human disease: an overview. Methods Enzymol 186: 1-85, 1990.

4. Dargel R: Lipid peroxidation - a common pathogenetic mechanism? Exp Toxicol Pathol 44: 169-181, 1992.

5. Kehrer JP and Smith CV: Free radicals in biology: sources, reactivities, and roles in the etiology of human diseases. In: Natural Antioxidants in Human Health and Disease. Frei B (ed). Academic Press, San Diego, CA, pp25-62, 1994.

6. Bors W, Michel C and Stettmaier K: Antioxidant effects of flavonoids. Biofactors 6: 399-402, 1997.

7. Halliwell B: Drug antioxidant effects. A basis for drug selection? Drugs 42: 569-605, 1991.

8. Ahmed AS, Nakamura N, Meselhy MR, Makhboul MA, El-Emary N and Hattori M: Phenolic constituents from Grevillea robusta. Phytochemistry 53: 149-154, 2000.

9. He QQ, Liu MS, Jin DJ and Kong LY: Phenolic glycosides from leaves of Hopiciopsis lobata. J Asian Natur Prod Res 8: 373-377, 2006.

10. Adisa RA, Abass Khan A, Oladosu I, Ajaz A, Choudhary MI, Olorunsogo OO and Ur Rahman A: Purification and characterization of phenolic compounds from the leaves of Cnestis ferruginea (De Candolle): Investigation of antioxidant property. Res J Phytochem 5: 177-189, 2011.

11. Boakye-Yiadom K and Konning GH: Incidence of antibacterial activity in the Connaraceae. Planta Med 28: 397-400, 1975.

12. Akiu S, Suzuki Y, Fujinuma Y, Asahara T and Fukada M: Inhibitory effect of Arbutin on melanogenesis: Biochemical study in cultured B16 cells and effect on the UV-induced pigmentation in human skin. Proc Jpn Soc Invest Dermatol 12: 138-139, 1988 (In Japanese).
13. World Health Organization: Cause-specific mortality and morbidity. http://www.who.int/whosis/whostat/EN_WHS09_ Table2.pdf. Accessed July 10, 2013.

14. Ferlay J, Shin HR, Bray F, Forman D, Mathers C and Parkin DM: Estimates of worldwide burden of cancer in 2008: GLOBOCAN 2008. Int J Cancer 127: 2893-2917, 2010.

15. Grever MCB: Cancer drug discovery and development. In: Cancer: Principles and Practice of Oncology. De Vita VT Jr, Hellman S and Rosenberg SA (eds). Lippincott Raven, Philadelphia, PA, pp328-339, 2001.

16. Peters GJ, Backus HH, Freemantle S, Van Triest B, Codacci-Pisanelli PG, et al: Induction of thymidylate synthase as a 5-fluorouracil resistance mechanism. Biochim Biophys Acta 1587: 194-205, 2002.

17. Desagher S and Martinou JC: Mitochondria as the central control point of apoptosis. Trends Cell Biol 10: 369-377, 2000.

18. Bernardi P: Mitochondrial transport of cations: channels, exchangers and permeability transition. Physiol Rev 79: $1127-1155,1999$.

19. Crompton M: The mitochondrial permeability transition pore and its role in cell death. Biochem J 341: 233-249, 1999.

20. Halestrap AP, McStay GP and Clarke SJ: The permeability transition pore complex: another view. Biochimie 84: 153-166, 2002.

21. Rasola A, Sciacovelli M, Pantic B and Bernardi P: Signal transduction to the permeability transition pore. FEBS Lett 584: 1989-1996, 2010.

22. Fadeel B, Orrenius S and Zhivotovsky B: Apoptosis in human disease: a new skin for the old ceremony? Biochem Biophys Res Commun 266: 699-717, 1999.

23. Endrini S, Jaksa S, Marsiati H, Othman F and Rahmat A: Effects of cola nut (Cola nitida) on the apoptotic cell of human breast carcinoma cell lines. J Medicinal Plant Res 5: 2393-2397, 2011.

24. Martin KR: Targeting apoptosis with dietary bioactive agents Exper Biol Med (Maywood) 231: 117-129, 2006.

25. Berridge MV, Herst PM and Lawen A: Targeting mitochondrial permeability in cancer drug development. Mol Nutr Food Res 53: 76-86, 2009.

26. Salako TA, Adisa RA, Alao OO, Adeniran OO, Atanu FO and Olorunsogo OO: Effects of methanolic and chloroform extracts of leaves of Alstonia boonei on rat liver mitochondrial membrane permeability transition pore. Afr J Med Med Sci 39 (Suppl 1): 109-116, 2010.

27. Johnson D and Lardy H: Isolation of liver or kidney mitochondria. Methods Enzymol 10: 94-96, 1967.

28. Lowry OH, Rosenbrough NJ, Farr AL and Randall RJ: Protein measurement with Folin phenol reagent. J Biol Chem 193: 265-275, 1951.

29. Varshney R and Kale RK: Effect of calmodulin antagonists on radiation induced-lipid peroxidation in microsomes. Internatl $\mathrm{J}$ Radiat Biol 58: 733-743, 1990.

30. Farombi EO, Ogundipe OO, Samuel Uhunwangho E, Adeyanju MA and Olarenwaju Moody J: Antioxidant properties of extracts from Alchornea laxiflora (Benth) Pax and Hoffman. Phytother Res 17: 713-716, 2003.

31. Lapidus RG and Sokolove PM: Inhibition by spermine of the inner membrane permeability transition of isolated rat heart mitochondria. FEBS Lett 313: 314-318, 1992.

32. Shawi AA, Rasul A, Khan M, Iqbal F and Tonghui M: Eupatilin: A flavonoid compound isolated from the artemisia plant induces apoptosis and G2/M phase cell cycle arrest in human melanoma A375 cells. Afr J Pharm Pharmacol 5: 582-588, 2011.

33. Nugraheni M, Santoso U, Suparmo H and Wuryastuti H: Potential of Coleus tuberosus as an antioxidant and cancer chemoprevention agent. Int Food Res J 18: 1471-1480, 2011.

34. Choi S, Jang HJ, Choi JY, Kim MS, Lee YR, Kim HS, et al: Antioxidant and anticancer activity of fractions of the ethanol extracts of Naematoloma sublateritium. J Med Plant Res 6: 1344-1352, 2012.

35. Stadtman ER: Metal ion-catalyzed oxidation of proteins: biochemical mechanism and biological consequences. Free Radical Biol Med 9: 315-325, 1990.

36. Lapidus RG and Sokolove PM: Spermine inhibition of the permeability transition of isolated rat liver mitochondria: an investigation of mechanism. Arch Biochem Biohphys 306: 246-253, 1993. 